Émergence du Vernaculaire en Europe

2| 2015

Homère en Europe à la Renaissance. Traductions et réécritures

\title{
Le mythe renaissant de Mars par ses épithètes
}

\section{Monica Barsi}

\section{(2) OpenEdition}

Journals

Édition électronique

URL : https://journals.openedition.org/eve/1256

DOI : $10.4000 /$ eve. 1256

ISSN : 2425-1593

Éditeur :

Université de Savoie, Université Jean Moulin - Lyon 3

Référence électronique

Monica Barsi, «Le mythe renaissant de Mars par ses épithètes », Corpus Eve [En ligne], 2 | 2015, mis en ligne le 31 décembre 2015, consulté le 28 avril 2022. URL : http://journals.openedition.org/eve/1256 ;

DOI : https://doi.org/10.4000/eve.1256

Ce document a été généré automatiquement le 28 avril 2022.

(c) Tous droits réservés 


\title{
Le mythe renaissant de Mars par ses épithètes
}

\author{
Monica Barsi
}

\section{NOTE DE L'AUTEUR}

Pour cette contribution, je n'ai malheureusement pas profité d'un ouvrage fondamental qui a paru ultérieurement et que je tiens à signaler : L'épithète, la rime et la raison. La lexicographie poétique en Europe, XVI ${ }^{e}$-XVII ${ }^{e}$ siècles, sous la direction de Sophie Hache et Anne-Pascale Pouey-Mounou, Paris, Classiques Garnier, 2015. Parmi les études, celle du regretté Philippe Ford, «Les épithètes homériques source d'embarras pour les premiers traducteurs » (p. 123-139), est particulièrement intéressante pour mon sujet.

1 Au cours du XVI ${ }^{e}$ siècle, en France, les allusions à Mars sont courantes et topiques. L'exemple de Guillaume des Autels est dans ce sens significatif. Il donne, dans son Eloge de la guerre, une image du dieu focalisée sur ses actions destructives qui s'accumulent et devraient même se multiplier suivant son exhortation :

\footnotetext{
Là quatre chevaulx roux tiroyent en grand vitesse

Le chariot sanglant de Mars, qui en la presse

Rompt, froisse, frappe, abbat de sa meurtriere main

Et ne se peult jamais souler du sang humain :

Ains tousjours en tuant, d'une voix effroyable

Exhorte tout le monde, à faire le semblable ${ }^{1}$.
}

2 Sans se référer à l'intégralité des allusions à Mars remontant à la période choisie, l'actualisation du mythe dans la production poétique en vernaculaire hérite d'une longue tradition d'origine latine et d'un certain nombre d'éléments novateurs provenant de l'Iliade qui circule dans différentes éditions tant en latin qu'en grec ${ }^{2}$. Cette contribution, qui n'est qu'une première étape de la recherche, portera sur les épithètes utilisées pour essayer de décrire les multiples aspects de la personnalité martiale. Les ouvrages du corpus isolé appartiennent à trois différentes catégories: la première, comprend des dictionnaires, ceux de Jean Tixier de Ravisi, de Charles Estienne et de 
Maurice de La Porte ; la deuxième, les traductions en français de l'Iliade, celle du latin de Valla de Jean Samxon et celle du grec de Hugues Salel et Amadis Jamyn ; la troisième, la poésie de Ronsard, lecteur éminent d'Homère. Les mots sélectionnés dans le vocabulaire définissant le mythe ont été répartis dans des regroupements onomasiologiques permettant de comparer plus facilement les éléments récurrents.

Dans l'Epithetorum opus absolutissimum de Jean Tixier de Ravisi ${ }^{3}$, plus d'une centaine d'adjectifs qualifient Mars. Ce foisonnement lexical atteste l'emploi non seulement très varié du mythe mais aussi sa signification, qui dans chaque contexte présente une acception différente. Le dieu est défini d'après des caractéristiques qui indiquent alternativement des traits reconnaissables quant à :

1) sa provenance: il est bistonius, geticus, odrysius, quirinus, strymonius sythonius, threicius ;

2) sa condition : il est armiger, armipotens, armisonans, armisonus, egregius, ensifer, inclytus, indomitus, invictus ;

3) son aspect: il est fortis, horrendus, horridus, horrificus, immanis, sanguineus, terribilis, torvus;

4) son caractère et son comportement : il est acer, amarus, ambiguus, anceps, asper, atrox, audax, bellifer, belliger, caecus, crudus, cruentus, deploratus, dubius, durus, efferus, ferox, ferreus, ferus, fervens, fremens, fulmineus, funestus, furens, generosus, homicida, hostilis, immitis, impavidus, impius, implacabilis, improbus, incertus, infaustus, infestus, infidus, inhumanus, iniquus, insanus, lascivus, magnanimus, nefandus, potens, rabidus, rapax, rigidus, scevus, severus, superbus, tristis, truculentus, turbidus, vagus, velox, vesanus, violentus.

Ces définitions signalent que Mars provient de la Thrace, combat avec des armes, effraie par son aspect, exerce plusieurs formes de cruauté, raisonne sans cohérence, agit toujours pour détruire son ennemi. Les mots dérivant de bellum sont nombreux et renvoient aux fonctions du dieu: bellator, bellicus, belliger, bellifer, bellipotens; sa personnalité et son action sont en effet directement liées et ne se rapportent qu'à la guerre. La personnalité du dieu est dominée, à quelques exceptions près, par une force destructive qui s'impose au-delà de toute nécessité et possibilité d'explication. Deux adjectifs seulement s'écartent de l'ensemble : generosus et magnanimus réorientent le mythe vers une dimension positive.

5 Le Dictionarium historicum ac poeticum ${ }^{4}$ de Charles Estienne répertorie une image du dieu où plusieurs couches temporelles se superposent. Le lexicographe rapporte l'origine $\mathrm{du}$ nom de Mars en s'appuyant sur Varron ; sa naissance, en remontant à l'union de Jupiter et Junon ou à la seule Junon aidée par Flore; son adultère avec Vénus et le stratagème de Vulcain; l'existence de sa sœur Bellone; ses animaux sacrés; le sens propre de l'adjectif martial, attribué aux sacerdoces, l'extension du sens de Martius (-ia, -ium) signifiant tant bellicosus que bellicus; son association à la planète. L'empreinte de la tradition latine est très forte mais, d'un point de vue linguistique, les deux synonymes de martius, bellicosus et bellicus, dénotent la prédisposition à la guerre qui est celle de ses origines. Les deux mots expriment en effet à partir du même étymon l'union constante entre ce qui a trait à la guerre et ce qui est le propre de la guerre.

6 Le recueil des Epithètes de Maurice de La Porte ${ }^{5}$ permet d'avoir un aperçu de la réception, dans la poésie française, du mythe où la valeur métonymique du mot Mars continue d'être employée, comme le lexicographe l'indique: "souventesfois ce mot [Mars] est prins pour la guerre ». En regroupant les adjectifs relevés par La Porte dans les catégories choisies, Mars est selon: 
1) sa condition : bellique, dieu vengeur, ferré, guerrier, port'-espee;

2) son aspect : effroiable, ensanglanté, fort, horrible, puissant ;

3) son caractère et son comportement: aime-sang, ardent, aspre, audacieus, aveugle, belliqueus, cholere, courageus, cruel, desdegneus, dommageable, douteus, enragé, felon, fier, forcené, foudroiant, furibond, furieus, genereus, hardi, homicide, imperieus, implacable, impetueus, impitoiable, indomtable, ineffroiable, inexorable, inhumain, insensé, invincible, ireus, magnanime, meschant, odieus, ravissant, rigoureus, ruineus, sanglant, sanguinaire, seditieus, severe, superbe, terrible, triste, tumultueus, turbulent, vagabond, violent.

7 L'article contient des mots provenant surtout de la tradition latine parmi lesquels l'on note les mots belliqueus et bellique qui confirment la nature primaire du dieu. De nombreuses correspondances subsistent entre les termes latins enregistrés par Tixier de Ravisi et les termes français. Par exemple, le latin virgilien coecus est traduit, de manière directe, par le français aveugle. Des termes tout nouveaux sont également introduits, comme par exemple félon, employé entre autres par Ronsard. D'autre part, certaines qualités d'ordre moral apparaissent lorsque le dieu est, au milieu de la bataille, genereux et magnanime, comme on l'a déjà observé, ou audacieux et courageux. Le répertoire de La Porte atteste de fait un enrichissement que le passage au vernaculaire a entraîné.

8 Les traductions en français de l'Iliade apportent des éléments oubliés de l'Arès homérique. Dans la traduction de Samxon ${ }^{6}$, Mars se présente avec différentes caractéristiques qui concernent :

1) sa condition : [avec Minerve, l'un des deux] chefz principaulx de bataille (XIII, f. 139r), dieu de bataille (XXI f. 191v-192r), dieu et arbitre des batailles (V, f. 57v), president des batailles (V, f. 58r);

2) son aspect : grant (XI, f. 125r, XIII, f. 143r), plain de opprobre (V, f. 69v), plain de sang (V, f. 56r) ;

3) son caractère et son comportement: convoiteux de tuer et ocire $(\mathrm{V}, \mathrm{f} .69 \mathrm{~V})$, cruel $(\mathrm{V}$, f. 56r et V, f. 69v), fascheux et ennuyeux (V, f. 69v), fol et insensé (XV, f. 154v), fol et inscavant (XXI f. 191v-192r), impacient de la douleur qu'il sentoit (V, f. 69v), immemoratif (V, f. 69v) indifferent (VIII, f. 92v), mauvaiz chief de courage (V, f. 69v), mortifere (IV, f. 53r), plain de folie $(\mathrm{V}, \mathrm{f} .69 \mathrm{v})$, plain de vanterie $(\mathrm{V}, \mathrm{f} .69 \mathrm{v})$, sanguinaire $(\mathrm{V}, \mathrm{f} .56 \mathrm{r})$, variable $(\mathrm{V}$, f. 69v).

Dans la traduction de Samxon, l'aspect de Mars suscite des sentiments de répulsion. Le mot bataille est au cœur du complément qui spécifie le domaine où Mars opère : chef principal de bataille, dieu de bataille, dieu et arbitre des batailles, president des batailles. La construction avec plain de est récurrente et sert à introduire des substantifs différents qui caractérisent à chaque fois le dieu : plain de sang, plain de opprobre, plain de vanterie, plain de folie. Plusieurs doublets synonymiques sont utilisés pour satisfaire le goût du lectorat de l'époque. Suivant un procédé courant, dans les constructions fol et insensé et fol et inscavant, le terme générique fol est précisé par le deuxième terme insensé et inscavant. Le manque de sens et de savoir est effectivement un trait propre au dieu Mars (souvent opposé à sa sœur Minerve). Le terme convoiteux suivi des deux verbes synonymiques tuer et ocire introduit la dimension du désir et de la jouissance qui vient s'ajouter sous forme d'une tendance irrépressible. Pour son caractère, il manifeste, en plus de la violence, la propension à se vanter et à être lâche, comme la locution mauvaiz chief de courage l'indique. Cette dimension, qui compromet la figure du guerrier, et encore plus celle du héros qui ne change jamais de camp même au prix de sa vie, 
correspond littéralement aux termes latins de la version de Valla animi malignum caput ${ }^{7}$. Cette dégradation est de toute évidence réintroduite dans la connaissance du mythe par la lecture de l'Iliade lorsqu'elle sera faite à partir du texte grec.

L'Arès homérique fait son apparition de manière frappante avec les traductions de Salel et Jamyn de l'Iliade ${ }^{8}$, qui introduisent des mots connus et nouvellement forgés. Le dieu est décrit suivant :

1) sa condition : bateur de boucliers martelez (XXI, f. 352r), immortel (XX, f. 338r), maudict $(\mathrm{V}, \mathrm{f} .85 \mathrm{v})$, mobile en son armet (XX, f. 330v), souillé de carnage (XXI, f. 352v);

2) son aspect: abattu (XXI, f. 352v), cruel (V, f. 82v), dolent (V, f. 81v), ennuyé (V, f. 71r), sain $(\mathrm{V}, \mathrm{f} .86 \mathrm{r})$, sanguinolent $(\mathrm{V}, \mathrm{f} .74 \mathrm{r})$, terrible $(\mathrm{V}, \mathrm{f} .74 \mathrm{r})$;

3) son caractère et son comportement : baveux $(\mathrm{V}, \mathrm{f} .84 \mathrm{r})$, causeur $(\mathrm{V}, \mathrm{f} .84 \mathrm{r})$, dangereux $(\mathrm{V}, \mathrm{f} .86 \mathrm{r})$, execrable $(\mathrm{V}, \mathrm{f} .85 \mathrm{v})$, fol inconstant $(\mathrm{V}, \mathrm{f} .84 \mathrm{v})$, furieux $(\mathrm{V}$, f. $74 \mathrm{r}$; f. 78r ; V, f. 83v ; $\mathrm{V}, 84 \mathrm{v})$, invincible $(\mathrm{V}, \mathrm{f} .74 \mathrm{r})$, malicieux $(\mathrm{V}, \mathrm{f} .85 \mathrm{v})$, mauvais $(\mathrm{V}, \mathrm{f} .86 \mathrm{r})$, menteur $(\mathrm{V}, \mathrm{f} .84 \mathrm{r})$, pernicieux (XXI, f. 353r), plein d'audace (XXI, f. 352v), triste (V, f. 85v), vanteur (V, f. 84r), variable (V, f. 84r-v ; V, f. 85v), violent (XX, f. 331v).

Certaines épithètes formulaires sont reproduites au pied de la lettre (c'est l'option de Jamyn) sans que leur construction soit lexicalisée: mobile en son armet

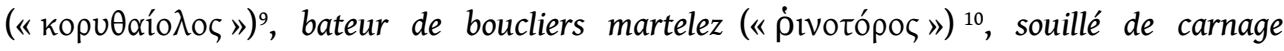

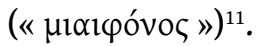

12 Les autres adjectifs appartiennent à un vocabulaire courant qui permet de saisir la nature du dieu caractérisé par des traits relevant non seulement de la violence comme force incontrôlée, mais aussi d'une certaine machination. Le terme malicieux indique sans aucun doute une composante rationnelle qui va au-delà de la spontanéité et de la contingence du moment $t^{12}$.

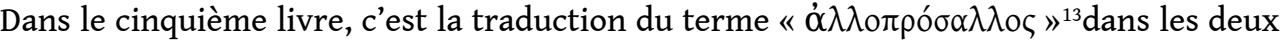
passages où il figure dans le texte grec qui introduit l'un des aspects les plus scabreux au sein de la dégradation de la valeur guerrière. La traduction de Salel a bien suivi sa source :

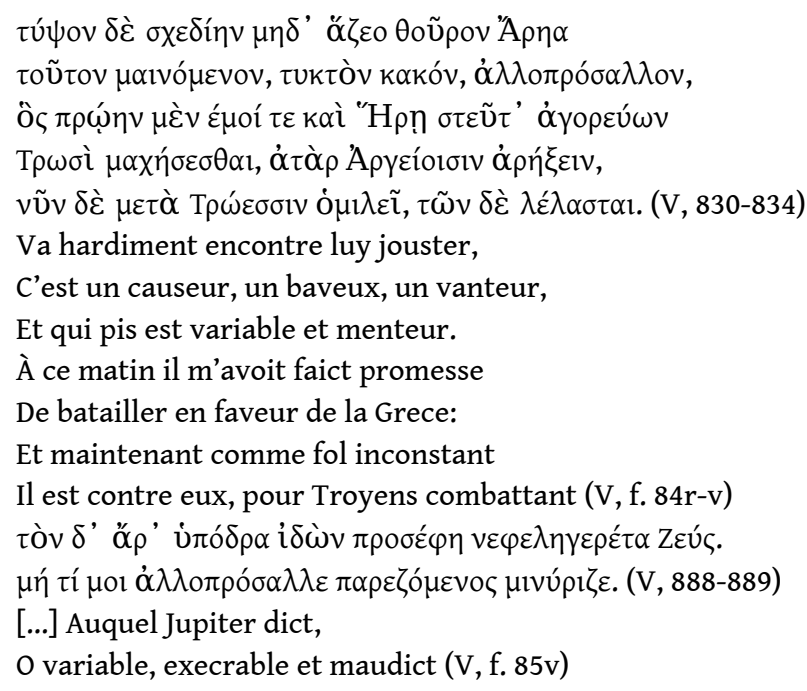

La réaction d'un des lecteurs de l'Iliade dans cette traduction nous permet de saisir l'effet suscité par cette épithète homérique, réservée uniquement à Arès. Dans son Tableau de l'inconstance et instabilité de toutes choses de 1607, Pierre de L'Ancre, magistrat à Bordeaux, commente le terme grec renvoyant à la variabilité du dieu : 
Et ne se prend pour la guerre [Mars], que d'autant qu'il aime le trouble, le sang \& la

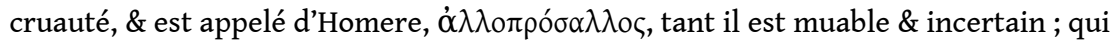
veut dire tantost à l'un, tantost à l'autre ; estant si pusillanime, si peu genereux et constant (tout Dieu qu'il est) qu'il se laissa blesser à Dionysius [sic pour Dyomède] homme mortel. Et Homere le feint si poltron, qu'il ne se peut deffendre contre Pallas, qui n'est qu'une femmelette. Si bien qu'en le desprisant à cause de sa lascheté, elle le dechire ainsi, \& publie son inconstance parlant à Diomedes :

C'est un causeur, un baveux, un vanteur,

Et qui pis est variable et menteur.

À ce matin il m'avoit fait promesse

De batailler en faveur de la Grece:

Et maintenant comme fol inconstant

Il est contre eux, pour Troyens ${ }^{14}$.

Dans ce commentaire, une série d'autres adjectifs contribuent à définir le dieu qui devient même pusillanime et poltron d'après un constat qui ne se réfère plus aux faits, mais à une autre échelle de valeurs qui atteste l'intégration toute naturelle du récit dans la culture d'arrivée.

En prenant en considération l'œuvre de Ronsard, le corpus que l'on peut extraire est significatif ${ }^{15}$. Par rapport au mythe du dieu guerrier, ses différentes allusions confirment sa référence à l'Iliade : Ronsard évoque Mars avec ses armes - la lance, la hache et le bouclier (Lm VIII, p. 191-193) - et avec ses chevaux - les roussins Thraciens (Lm XV, p. 145), il le décrit lorsqu'il se bat avec Minerve (Lm XV, p. 133), lorsqu'il est blessé par Diomède (Lm VI, p. 32) et lorsqu'il est prisonnier des Aloades (Lm VIII, p. 81). En ce qui concerne le vocabulaire qui sert à décrire le dieu, Mars est caractérisé par :

1) son aspect : couvert de sang, de discord et de noise (Lm XVII, p. 351), jeune (Lm XII, p. 77), horrible (Lm III, p. 91 ; V, p. 175), horrible d'une grande creste (Lm XV, p. 347), renfrongné d'une ire noire (Lm III, p. 132), tout sanglant (Lm VI, p. 206) ;

2) son caractère et son comportement: cruel (Lm IX, p. 136; p. 167), furieus (Lm XV, p. 133), menaçant et grondant (Lm I, p. 28), meurdrier (Lm XV, p. 348), puissant (Lm VIII, p. 64), tout alumé de rage (Lm XV, p. 133), tout colere (Lm VIII, p. 30), tout fellon (Lm XV, p. 348), volage (Lm, V, p. 216), vomissant sa fureur (Lm III, p. 4).

Le vocabulaire dont le poète se sert comprend surtout des syntagmes, produits par une créativité lexicale fondée sur certains stylèmes, mais aussi sur les surprises de la variation.

Dans le doublet menaçant et grondant, le premier terme annonce le second, plus précis. Le terme spécifique grondant explicite la forme de la menace et renvoie aux cris de Mars lorsqu'il est blessé par Diomède: "il crie comme le feraient neuf ou dix mille combattants » (Il., V, 1142-1143). En effet, le son inarticulé, qui s'oppose à la voix en tant qu'expression de la culture et de l'éducation, représente toujours une menace. Dans la traduction de Salel, les adjectifs qui se réfèrent aux bruits de Mars ou en présence de Mars expriment toujours un danger. Dans le cinquième livre, ils sont par exemple associés à l'adjectif épouvantable ${ }^{16}$, alors que le vacarme de Mars et Pallas devient par synesthésie sanglant ${ }^{17}$. Le doublet créé par Ronsard semble donc bien reproduire l'état d'âme provenant du danger perçu par l'ouie.

Un autre exemple est le syntagme vomissant sa fureur et l'aspre venin de sa rage. L'agressivité du dieu est expliquée par la fureur et la rage qui l'animent; le participe présent vomissant marque la portée de l'action par sa contingence et s'accompagne de deux compléments d'objet direct où le mot venin et le mot rage rappellent deux 
locutions : vomir venin et vomir rage. De plus, le double complément sa fureur et l'aspre venin de sa rage reproduit la structure du doublet où la fureur est l'aspre venin de sa rage.

En ce qui concerne la vue, deux syntagmes représentent l'aspect effroyable du dieu. Dans la qualification renfrongné d'une ire noire, le complément indique la cause et associe la vue (la grimace du visage) au sentiment (l'ire) renforcé par le sens métaphorique attribué à la couleur (le noir). La construction horrible d'une grande creste contient l'adjectif horrible qui renvoie à l'aspect effrayant du dieu et qui est expliqué par le mot creste. Le casque est depuis toujours l'un des éléments de l'armature qui suscite, au niveau visuel, la crainte (cet élément est d'ailleurs récurrent dans la description des combattants de l'Iliade, Hector en est un exemple).

Dans d'autres cas, Ronsard rappelle certaines caractéristiques du dieu en les décrivant. Ses dimensions sont par exemple surhumaines : « [il] Passe du doz et de toute la face / Les chevaliers qu'il ameine de Thrace. ${ }^{18}$ ”

Dans l'Hymne de Tresillustre Prince Charles Cardinal de Lorraine, après la défaite de Saint Quentin (août 1557), c'est l'inconstance du dieu qui est évoquée :

Au temps le plus cruel, quand le sort hazardeux

De Mars, qui la victoire aux Princes oste et donne,

Luy esbranla des mains le sceptre et la couronne (Lm IX, p. 50, v. 380-382).

Des liens plus subtils renvoient encore à l'Iliade. En se référant à Mars pour rendre hommage à Anne de Montmorency, Ronsard compare le connétable au dieu :
Dans le meillieu du Temple Anne Mommorency
Sera portrait seul, mais portrait tout ainsi
Qu'un Mars est equippé, quand il arenge en armes
Du long bout de sa pique un peuple de gendarmes,
Ou quand il pousse à-bas les murs d'une cité,
Contre les citoyens justement irrité.
Ou pource qu'ilz n'ont pas aux paouvres fait justice,
Ou qu'ilz n'ont pas aux Dieux payé leur sacrifice ${ }^{19}$.

La proposition temporelle "quand il pousse à-bas les murs d'une cité » est vraisemblablement une allusion au dieu qui, d'après l'une de ses épithètes formulaires,

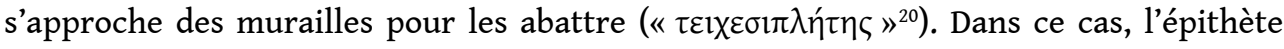
serait défigée et réemployée dans une phrase où, toutefois, l'adverbe justement modulant l'adjectif irrité modifie le sens de ce mot en lui conférant une valeur positive indiscutable. Cette précision rappelle, bien qu'indirectement, un autre texte qui faisait partie du corpus homérique, c'est-à-dire l' Hymne à Arès où Arès garantit l'accomplissement de la justice.

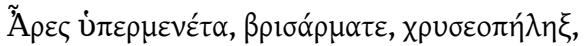

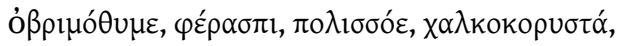

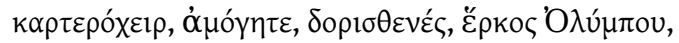

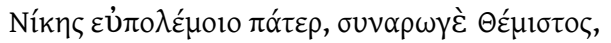

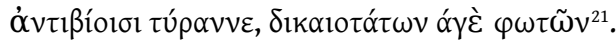

Dans tous les cas mentionnés, la création poétique offre, grâce à l'adaptation linguistique, la possibilité d'accéder à un univers inconnu qui devient ainsi familier.

Étant donné la présence constante de la guerre, la Renaissance revisite, par l'arrivée de l'Iliade dans ses versions originelles, le statut archaïque du grec Arès pour lequel la prise en compte de l'autre fait encore partie d'une expérience agressive et éloignée de toute instance civilisatrice. Cette primordialité qui est à la base de la naissance d'un dieu guerrier plutôt que d'un dieu de la guerre, c'est-à-dire une entité meurtrière en- 
deçà d'une intelligence stratégique, est comprise comme un trait très humain. Par la double traduction de la lettre et du sens, le récit homérique enrichit, à travers un jeu de renvois et d'interprétations, le vocabulaire mettant en scène le premier guerrier de tous les temps. Tout relevé linguistique permet d'observer comment les expressions homériques s'intègrent au patrimoine lexical disponible, provenant en grande partie du fonds latin, et comment elles renouvèlent le mythe. La recherche qui a été proposée au cours de la journée d'étude dont les actes sont ici réunis nous suggère la possibilité de comparer ces procédés d'assimilation dans différentes langues. Ce sera aussi l'occasion pour enrichir les dictionnaires historiques avec une banque de mots littéraires qui pourront aussi être rapprochés des mots du quotidien ou, comme dans ce cas, de ceux qui ont été utilisés dans tous les écrits qui ont trait à la guerre, et de dresser un bilan entre tradition et innovation.

\section{BIBLIOGRAPHIE}

DES AUTELS, Guillaume, Remonstrance au peuple Françoys, de son devoir en ces temps, envers la majeste du roy. A laquelle sont adjoustez troy Eloges, De la paix, De la trefve, et De la guerre, Paris, André Wechel, 1559.

ESTIENNE, Charles, Dictionarium historicum ac poeticum, omnia gentium, hominum, locorum, fluminum ac montium antiqua recentioraque, ad sacras ac prophanas historias poetarumque fabulas intelligendas necessaria, vocabula... complectens, a Carolo Stephano, illius authore... adauctum, Lutetiae, J. Macé, 1561. FORD, Philip, De Troie à Ithaque : réception des épopées homériques à la Renaissance, Genève, Droz, 2007. FORD, Philip, «Les épithètes homériques source d'embarras pour les premiers traducteurs ", in L'épithète, la rime et la raison. La lexicographie poétique en Europe, $\mathrm{XVI}^{e}-\mathrm{XVII}{ }^{e}$ siècles, dir. Sophie HACHE et Anne-Pascale POUEY-Mounou, Paris, Classiques Garnier, 2015, p. 123-139.

HEPP, Noémi, Homère en France au XVI esiècle, Atti della Accademia delle Scienze di Torino, vol. 96, 1961-62, p. 383-508.

HOMÈRE, Homeri, poetce clarissimi, Ilias, per Laurentium Vallensem,... e greco in latinum translata et nuper accuratissime emendata, Lipsiæ, Melchiorem Lotterum, 1512.

HOMÈRE, Les Iliades de Homere, Poete Grec et grant hystoriographe, Avecques les Premisses et commencemens de Guyon de Coulonne souverain hystoriographe. Additions et sequences de Dares Phrigius et de Dictys de Crete. Translatees en partie de latin en langaige vulgaire Par maistre Jehan Samxon licentié en Loys Lieutenant du Bailly de Touraine à son siege de Chastillon sur Yndre, Paris, Jehan Petit, 1530.

HOMÈRE, Les XXIIII Livres de l'Iliade d'Homere, princes des Poëtes Grecs. Traduicts du Grec vers François. Les XI. premiers par M. Hugues Salel Abbé de Sainct Cheron et les XIII. Derniers par Amadis Jamyn, Secretaire de la chambre du Roy : tous les XXIIII. reveuz \& corrigez par ledit Am. Jamyn avec Le premier \& second de l'Odyssée d'Homere, par Jaques Peletier du Mans. Plus une table bien ample sur l'Iliade d'Homere, Paris, Lucas Breyer Libraire, 1580.

HOMÈRE, Hymnes, texte établi et traduit par Jean Humbert, Paris, Les Belles Lettres, 1976. 
L'ANCRE, Pierre de, Tableau de l'inconstance et instabilité de toutes choses, Paris, Abel L'Angelier, 1610.

L'épithète, la rime et la raison. La lexicographie poétique en Europe, $\mathrm{XVI}^{e}-\mathrm{XVII}{ }^{e}$ siècles, dir. Sophie HACHE et Anne-Pascale POUEY-MOUNOU, Paris, Classiques Garnier, 2015.

LA PORTE, Maurice de, Les Épithètes, Paris, G. Buon, 1571.

PRIETO, María Luz, Ares en Homero: Función del dios de la guerra en la Ilíada y la Odisea, Amsterdam, A. M. Hakkert, 1996.

RONSARD, Pierre de, Euvres complètes, éd. critique, avec introduction et commentaire par Paul Laumonier, Paris, Société des textes français modernes, 8 vol., 1914-1919.

SPONDE, Jean de, Homeri qua extant omnia, Ilias, Odyssea, Batrachomyomachia, Hymni, proematia aliquot, cum latina versione omnium quœe circumferuntur emendatiss. aliquot locis jam castigatiore, Basileæ, Eusebii Episcopii Opera, 1583.

TIXIER DE RAVISI, Jean, Epitheta Joannis Ravisii Textoris,... quibus accesserunt De Prosodia libri IIII, quos Epithetorum prceposuimus operi. Item de Carminibus ad veterum imitationem artificiose componendis praecepta, collecta a Georgio Sabino..., Lugduni, N. Buon, 1616.

\section{NOTES}

1. Guillaume Des Autels, Remonstrance au peuple Françoys, de son devoir en ces temps, envers la majeste du roy. A laquelle sont adjoustez troy Eloges, De la paix, De la trefve, et De la guerre, Paris, André Wechel, 1559 , in-4, f. $12 v$.

2. Sur ces ouvrages, voir les contributions fondamentales de Noémi Hepp, Homère en France au XVI siècle, Atti della Accademia delle Scienze di Torino, vol. 96, 1961-62, p. 383-508 et de Philip Ford, De Troie à Ithaque : réception des épopées homériques à la Renaissance, Genève, Droz, 2007.

3. Epitheta Joannis Ravisii Textoris,... quibus accesserunt De Prosodia libri IIII, quos Epithetorum praeposuimus operi. Item de Carminibus ad veterum imitationem artificiose componendis praecepta, collecta a Georgio Sabino..., Lugduni, N. Buon, 1616, p. 552-554.

4. Dictionarium historicum ac poeticum, omnia gentium, hominum, locorum, fluminum ac montium antiqua recentioraque, ad sacras ac prophanas historias poetarumque fabulas intelligendas necessaria, vocabula... complectens, a Carolo Stephano, illius authore... adauctum, Lutetiae, J. Macé, 1561.

5. Maurice de La Porte, Les Épithètes, Paris, G. Buon, 1571 (sv. Mars).

6. Les Iliades de Homere, Poete Grec et grant hystoriographe, Avecques les Premisses et commencemens de Guyon de Coulonne souverain hystoriographe. Additions et sequences de Dares Phrigius et de Dictys de Crete. Translatées en partie de latin en langaige vulgaire Par maistre Jehan Samxon licentié en Loys Lieutenant du Bailly de Touraine à son siege de Chastillon sur Yndre, Paris, Jehan Petit, 1530.

7. Homeri, poetce clarissimi, Ilias, per Laurentium Vallensem,... e greco in latinum translata et nuper accuratissime emendata, Lipsiæ, Melchiorem Lotterum, 1512, f. XXVv.

8. Les XXIIII Livres de l'Iliade d'Homere, princes des Poëtes Grecs. Traduicts du Grec vers François. Les XI. premiers par M. Hugues Salel Abbé de Sainct Cheron et les XIII. Derniers par Amadis Iamyn, Secretaire de la chamber du Roy: tous les XXIIII. reveuz \& corrigez par ledit Am. Iamyn avec Le premier \& second de l'Odyssée d'Homere, par Iaques Peletier du Mans. Plus une table bien ample sur l'Iliade d'Homere, Paris, Lucas Breyer Libraire, 1580.

9. Il.XX 38.

10. Il. XXI 392. 
11. Il. XX 403. En ce qui concerne ce vocabulaire, je ne mentionne que la monographie de María Luz Prieto, Ares en Homero: Función del dios de la guerra en la Ilíada y la Odisea, Amsterdam, A. M. Hakkert, 1996.

12. Jean de Sponde condamne lui aussi le dieu en lui attribuant une perfidie qui semble expliquer son instabilité : " notanda est Martis perfidia, qui se Menelao favere simulat, dum tamen in eius perniciem conspirat [...] ausus sum hoc in Marte perfidiam appellare, quia illa ipsi sit familiarissima, unde $\dot{\alpha} \lambda \lambda$ 入 omnia, Ilias, Odyssea, Batrachomyomachia, Hymni, proematia aliquot, cum latina versione omnium qua circumferuntur emendatiss. aliquot locis jam castigatiore, Basileæ, Eusebii Episcopii Opera, 1583, p. 93.

13. Il. V 831, 889.

14. Cette citation est tirée de la deuxième édition de l'ouvrage: Pierre de L'Ancre, Tableau de l'inconstance et instabilité de toutes choses, Paris, Abel L'Angelier, 1610, f. $478 \mathrm{r}$ et v.

15. Nous nous référons à l'édition de Paul Laumonier : Pierre de Ronsard, Cuuvres complètes, éd. critique, avec introduction et commentaire par Paul Laumonier, Paris, Société des textes français modernes, 8 vol., 1914-1919.

16. « Mars furieux, et Bellone la forte / Marchoient devant la Troyenne cohorte. / Elles faisoient un bruit espouvantable, / Et Mars bransloit sa lance redoubtable » (V, f. 78r) ; « De ce grand coup et blessure notable / Mars fit un cry autant espouventable, / Et plus hideux que ne feroyent dix mille / Vaillans soldats assaillans une ville, / Dont les Troyens et Gregeois attentifs / Soudainement devindrent tous craintifs » (V, f. 85r), Les XXIIII Livres de l'Iliade d'Homere, princes des Poëtes Grecs. Traduicts du Grec vers François. Les XI. premiers par M. Hugues Salel Abbé de Sainct Cheron et les XIII. Derniers par Amadis Jamyn, éd. cit.

17. «Les deux Aiax soudain furent environnez / De bandes de Soldats à la guerre bien nez, / Bandes qui se montroient si vaillantes aux armes / Que ny Mars ny Pallas dieux des sanglants vacarmes / N'eussent pas mesprisé » (XIII, 127, p. 211r), Les XXIIII Livres de l'Iliade d'Homere, princes des Poëtes Grecs. Traduicts du Grec vers François. Les XI. premiers par M. Hugues Salel Abbé de Sainct Cheron et les XIII. Derniers par Amadis Jamyn, éd. cit.

18. Ronsard, Priere à la Fortune A tresillustre et reverendissime Cardinal de Chastillon, Lm VIII, p. 111, v. 215-216.

19. Ronsard, "Temple de Messeigneurs Le Connestable, et des Chastillons », Lm VIII, p. 74, v. 23-30.

20. Il. V 31 et 455.

21. «Arès souverainement fort, fardeau des chars, Dieu casqué d'or, cœur vaillant, toi qui sais porter le bouclier, protecteur des cités, tout revêtu d'airain, bras puissant, infatigable, fort par ta lance, rempart de l'Olympe, père de la Victoire qui clôt heureusement les guerres, soutien de la Justice, toi qui maîtrises l'adversaire et diriges les hommes les plus justes ", Homère, Hymnes, texte établi et traduit par Jean Humbert, Paris, Les Belles Lettres, 1976, 1-5. 


\section{RÉSUMÉS}

Cette contribution examine certaines épithètes attribuées à Mars à la Renaissance. Le corpus d'ouvrages pris en considération comprend les répertoires de Jean Tixier de Ravisi, de Charles Estienne et de Maurice de La Porte, les traductions en français de l'Iliade, celle du latin de Valla de Jean Samxon et celle du grec de Hugues Salel et Amadis Jamyn, ainsi que la poésie de Ronsard. Des mots littéraires et des mots plus quotidiens sont utilisés entre tradition et innovation, mais ce sont surtout les expressions homériques qui enrichissent le patrimoine lexical disponible, provenant en grande partie du fonds latin.

\section{INDEX}

Index géographique : domaine français

Index chronologique : XVIe siècle

Mots-clés : Mars, Arès, épithètes, lexicographie, traduction Iliade, français préclassique, Jean Samxon, Hugues Sales, Amadis Jamyn, Pierre de Ronsard

\section{AUTEUR}

\section{MONICA BARSI}

Monica Barsi est professeure de Langue française et de Didactique de la langue française à l'Università degli Studi di Milano. Ses études portent, en ce qui concerne l'histoire de la langue, sur le français préclassique et sur la lexicographie bilingue, et, en ce qui concerne l'histoire de l'enseignement, sur le français et l'italien comme langues étrangères. 